- Research in the general dental practice is facilitated by membership of a research network.

- A creative approach to the funding of a research network is needed.

- The level of research awareness among GDPs is already high and is readily translated into research activity by membership of a research network.

- General dental practitioners feel that research can enhance the delivery of oral care.

\title{
A general dental practice research network - philosophy, activities and participant views
}

\author{
E. J. Kay ${ }^{1}$ N. Ward ${ }^{2}$ and D. Locker ${ }^{3}$
}

The importance of building research capability among general dental practitioners was highlighted when in 1996 a large tranche of money was released to support research and development in primary dental care. As a result of the links forged by this research opportunity, a group of primary dental care practitioners was recruited into a Primary Dental Network. This network was closely allied to an academic department and the aim was to develop the practitioners' research interest into research skills and finally into a research project. The group therefore attended a series of structured workshops, designed specifically to augment the practitioners' skills using a 'learning by doing' approach. Once the practitioners had acquired the relevant skills, they undertook a research project, which will be reported in a later paper.

Through the Network's activity practitioners developed skills in literature retrieval, critical appraisal, measurement tools, data collection, research methodologies, protocol writing and data analysis. The practitioners were then asked for their views about and feelings towards their research network. This paper reports the thinking behind the research network and describes the workshops in detail. It also reports on the practitioners' views about their own experiences of the Network.

\section{INTRODUCTION}

Practice based research networks enable the study of primary care problems in a pri-

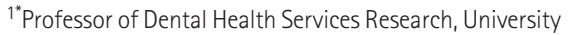
of Manchester Dental Hospital and School, Higher Cambridge Street, Manchester M15 6FH; ${ }^{2}$ Associate Postgraduate Dental Dean, Department of Postgraduate Medicine and Dentistry, Gateway House, Piccadilly South, Manchester M60 7LP; ${ }^{3}$ Director, Community Dental Research Unit, University of Toronto, Toronto, Canada To contact the North West Dental Practice Research Network this is c/o Gateway House, Gateway House, Piccadilly South, Manchester M60 7LP

${ }^{*}$ Correspondence to: E. Kay

E-mail:e.kay@man.ac.uk

\section{Refereed Paper}

Received 01.10.02; Accepted 04.03.03

$\odot$ British Dental Journal 2003; 194: 545-549 mary care setting. Whilst funding and support for such networks in medical primary care has been substantial, very few dental networks have been established.

The aims of the medical research networks, most of which were set up in 1998, were to:

1. Improve the quality of primary care research,

2. Increase research capacity in primary care, and

3. Change the culture of primary care towards reflective inquiring practice.

Dental Health Service research workers in the North West felt that such aims were as relevant to dentistry as to medicine and differed substantially from the objectives of networks which exist primarily in order to use the participants as a conduit for data collection rather than with the practitioners driving the research agenda.

The experience of primary care networks in general medical practice indicates that a number of key components are needed in order for a network to be successfully established and become research active. These characteristics include linkage with academic departments, an adequate mix of different types of practice, regular meetings and the appointment of a research fellow. ${ }^{1,2}$ Funding for the participants in order that their research activities do not undermine their income is also a prerequisite for a successful research network.

There is little doubt that primary care research networks can produce substantive research with direct applicability to dayto-day patient management. General medical practice research networks have investigated subjects as diverse as accuracy of data entry, ${ }^{3}$ appropriateness of out of hours calls, ${ }^{4}$ drug treatments for hypertension ${ }^{5}$ and recall intervals. ${ }^{6}$

What is common to all the research carried out by primary care research networks is that the objective of the research work is to address an issue which the involved practitioner feels is relevant to his or her practice. Thus, geographical and local factors affecting the participants' practice populations have major impact on the networks' research agenda. For example, an Oklahoma based network researched the diagnosis and treatment of recluse spider bites in the primary care setting $^{7}$ whilst another network compared the prevalence of alcohol abuse in two different populations. ${ }^{8}$ Thus, while 
the research results do not always have a major impact on the practice worldwide or directly benefit global populations, network research invariably addresses issues of real importance to practitioners and their own community of patients. Thus, a practitioner-driven focus for any research carried out is of profound importance to a research network in any discipline. The will to facilitate such research stimulated the setting up of the dental practice research network described in this paper. The research undertaken by the Dental Practice Research Network will be described in a subsequent communication.

\section{BACKGROUND}

In 1996, a tranche of funding for projects in Primary Dental Care was released by the Government. Involvement in the commissioning of projects led the authors of this paper to recognise that there was an acute need for research capability building, if primary care practitioners were to be successfully involved in research and development. It became apparent that although many general dental practitioners and community dental service personnel were willing to participate in research, often their role was diminished to data collection rather than them playing a full and active role in the development of research which addressed their own agendas. The first step in the project described here was the setting up of a research symposium for North West-based practitioners who wanted academic support for their bids to the Primary Dental Care R \& D Fund. At this symposium, there was abundant enthusiasm and willingness to participate in research among the practitioners. However, there was a paucity of research expertise, statistical and computer skills for analysis of data, plus a sense of isolation, poor understanding of funding processes and sense of 'distance' from academia. It was also noted that practitioners often felt that published research was not relevant to the day-to-day practice of dentistry, or to their patients. Many, although they would like to be involved in research, felt that their only potential role was as a 'datagatherer' for academics, whose research hypotheses were not necessarily considered relevant, interesting, or important, by practitioners of general dentistry.

However, as a direct result of the Primary Dental Care REtD Funding Initiative, links between academics and dental practitioners were forged. Further discussions with the Associate Postgraduate Dental Dean (Continuing Education) led to the development project described in this report. As far as can be discerned, there had not previously been a similar initiative.

The aim of the project was to support and develop practitioner research by developing dentists' ideas into projects, which would form the basis of a substantive research portfolio.

\section{METHOD}

Building and funding a research network

Building the research network consisted of a number of interdependent initiatives. Firstly, funding was sought for a post-doctoral researcher. It was envisaged that such an individual would attract funding to enable expansion and development of a Dental Research Network. Funding for such a researcher was achieved via the North West Regional Office.

Secondly, liaison with the Associate Postgraduate Dental Dean at the North West Deanery and involvement with the Faculty of General Dental Practitioners attracted financial support so that involved practitioners could be supported in part by the postgraduate training budget.

Thirdly, a successful bid was made to the North West Regional Office for funding for a Level Two Research Network. This was a funding stream for supporting general medical practitioner research networks but the scheme described here for general dental practitioners was considered worthy of financial support. This was because the special difficulties faced by dental practitioners wishing to undertake research in their practices were understood by the Regional Office, as it was they who had been responsible for the distribution of the Primary Dental Care Research and Development funding.

\section{Building research capability}

Research capability spans a range of skills and commitment ranging from 'research awareness', through 'research active' to 'academic'.

All practitioners probably have some level of research awareness. The practitioners in this Network, given that they signed up to belong to a research network, were not only research aware but wished to become research active. It had been clear from the symposium held by Manchester University to support GDPs bids for NHS RED funding, that most practitioners felt that they needed to enhance their research capabilities before they could become actively involved in research. A series of five workshops was therefore planned.

The aims of the workshops were twofold. The first aim was to develop practitioners' research skills in: literature retrieval, critical appraisal, design of measurement instruments/data collection tools, research methodologies, protocol development, data manipulation by computer and statistical analysis. The second aim was to support the development of the practitioners' own research ideas, with a view to the Network undertaking a research programme of their own devising. It was decided that this would best be achieved by the incremental development of research skills so that a series of workshops led to the discovery of issues, which were then addressed in subsequent workshops. By encouraging the practitioners to actually use and discuss what they had developed/ learnt in each workshop within their own practices, ideas for improvement and further research agendas were developed. This process is described in greater detail below.

\section{Workshop 1}

One of the key skills and a prerequisite to the development of any programme of research is the ability to quickly and efficiently search and obtain the relevant literature. Furthermore, once retrieved, if a case for a research idea is to be established taking current knowledge into account, the literature must be critically appraised, ie the practitioner must develop an 'eye' for good, robust research and for research which, although it may initially appear to be impressive, is actually fundamentally flawed.

The Cochrane Centre in Manchester is a repository of literature search and appraisal skills. The Network's workshop was therefore run by staff from the Cochrane Centre who led the practitioners through the process for finding and retrieving relevant research via computer searching. The workshop then analysed a research paper, dissecting out for the practitioners the key points to look for when reading the literature. This first workshop therefore developed the GDPs skills in literature searching and taught them exactly how to assess and judge the quality of research papers. Thus, whilst they developed their critical appraisal skills, they also gained an understanding of the profound importance to any piece of research of a robust methodology and an appropriate outcome measure. By the end of the workshop, the group were able to recognise strengths, weaknesses and potential confounding factors in published research. The practitioners also recognised that, unless there were already validated tools available to quantify the factors they wished to measure, the first step in research was to find, or develop outcome measures appropriate to the subject matter in hand. The importance of relevant, valid and reliable outcome measures in any study was a key issue in the GDPs' discussions. Indeed, one of the conclusions drawn by the Network practitioners at this workshop was that they did not consider many of the outcome measures documented in the literature as being relevant, applicable or usable in general dental practice research. 
This problem therefore underpinned the subject matter in the next workshop.

\section{Workshop 2}

Workshop 2 built on Workshop 1 and was designed to encourage the Network GDPs to form their own ideas for research in general practice. Taking forward the key issue raised in Workshop 1, the practitioners worked in pairs or groups of three. They were asked to try to determine a way of addressing the research issues they felt were important in relation to their role as practitioners. An extremely robust and sometimes heated debate as to the relevance of measures such as DMFT, plaque indices, CPITN, etc to general practice ensued. The practitioners drew the conclusion that the main concern of both themselves and their patients was that the treatment the patient received from the dentist 'worked', ie resulted in the patient perceiving some benefit. The practitioners were then given the task of both defining and planning how to measure whether a patient perceived benefit from treatment, ie whether the patient's life had been improved, or at least spared from further distress by the interventions undertaken by the dentists. This research agenda provided a forum for exploration of the problems associated with measuring "treatment impact' or 'health gain'. The practitioners felt strongly that the traditional clinical indices did not necessarily coincide with how the patient felt, nor did such indices reflect the quality of the oral health care the patients received.

The practitioners then went on to define the attributes of a patient's life which might be impacted upon by either their oral health status or by the quality of dental care the patient had received. Having delineated these attributes, the practitioners then identified the indicators of these attributes. For example, an important attribute of a healthy mouth was thought to be that it looks pleasant. An indicator of such an attribute would be the patient's level of satisfaction with their appearance. The Network practitioners then attempted to list items, which might be asked, in order to quantify the presence or absence of indicators in an individual person. For example, in the case of perception about appearance, the patient might be asked about the degree of embarrassment or shyness their appearance had caused them. Lastly, the practitioners undertook an item reduction exercise in which the practitioners chose between items which measured the same attribute.

A questionnaire was thus developed by the practitioners, with the assistance of an expert in the development of oral health impact outcome measures (Professor D
Locker, University of Toronto). The practitioners felt that the next step would be to pilot test the questionnaire in their practices. As part of the 'learning by doing' process, the questionnaire was duly distributed to practices for piloting with the Network practitioners' own patients.

\section{Workshop 3}

Workshop 3 involved the practitioners individually feeding back information regarding the piloting of the oral health impact questionnaire. A number of changes were considered to be important and some additions were made.

The dentists were then asked to consider how this recently developed instrument could be appropriately and usefully used in research in general practice. The practitioners felt that the instrument would be best used for the purpose for which it had been designed, ie to identify how dental care and treatment contributed to patients' feelings of health and well-being.

At this point, a research methodologist gave a presentation relating to study design. The strengths, weaknesses and difficulties involved in surveys, casecontrol, cohort intervention studies and randomised controlled trials were discussed. The GDPs then were asked to work in small groups. Each group was assigned one of the above research methodologies and was asked to design a practice-based study, utilising the piloted questionnaire as their main outcome measure. The Network was then taught the principles of protocol writing and then each subgroup developed their research 'plan' into a protocol. Each protocol was presented to the remainder of the group. This practical experience of protocol writing was a key skill developed among the Network practitioners.

\section{Workshop 4}

Workshop 4 drew on the previous three so that the research network could develop their own programme of dental primary care research to be conducted within their own dental practices. The practitioners, working as a group, chose one of the protocols (from Workshop 3) and developed it to describe a study in which they would wish to be involved. They designed a study which planned to measure:

1. The impact of oral health on their patient 2. Attributes of themselves and their practice which might affect patients' oral health impacts, and

3. (if funding allowed continuation of the Network) Evaluate the contribution to well-being provided by the oral care delivered by the dentists.

The practitioners decided that they should each randomly select 50 patients in each of their practices to take part in this multi-centre programme of research.

The research fellow then visited each practice and arranged data collection according to the protocol devised by the practitioners. Close liaison with the practice managers, receptionists and nursing staff was required and their input and cooperation was found to be crucial to the conduct and success of the study.

\section{Workshop 5}

Prior to the fifth workshop, the research fellow collected, collated and cleaned the data from the 15 dental practices which were participating in the data collection exercise. Each practice's data was put on to a floppy disk in SPSSX format. At the workshop, each practitioner was given his or her data disk, a computer workstation and a manual. A manual was produced by the Research Fellow, which took each practitioner through a set of exercises of increasing difficulty. It instructed them, in a step by step fashion, how to manipulate and analyse the data from their practice. The practitioners learned to label data, understand data coding, add variables to the dataset, calculate new data items from existing data, produce graphical outputs from numerical data, and illustrate the distribution of data.

Each practitioner completed 12 exercises with their data. Thus, this workshop introduced computer skills to those who did not already have them, taught data handling skills, and allowed the practitioners to view the data from their own practice and compare it visually and numerically with the data from other practices.

\section{Workshop 6}

Subsequent to the fifth workshop, a questionnaire measuring the practitioners' attitudes and 'practice style' was circulated to the Network members in order to address the second aim of the Network's study (see Workshop 4).

These data, plus the combined patient data collected from Workshop 5, were then analysed. Workshop 6 consisted of feeding the analysed results of the first two parts of the Network's study back to the practitioners. The workshop also conducted a feedback questionnaire, the results of which are described below. The results of the data analysis are described in a subsequent paper.

Finally, this workshop, being held as it was, near to the conclusion of the Network's funding, was used to plan for the future, discuss the Network's research agenda and agree how the data gathered could be best used.

There was a strong and unanimous feeling that the Network was extremely 
valuable, that the work undertaken was interesting, that the baseline research data were useful and that the Network should continue.

\section{THE PRACTITIONERS POINT OF VIEW} The experience of being in the network

A number of themes emerged in the Network practitioners' responses to 'write your comments about being in the Dental Practice Network'. These were: the importance of being part of a group, the educational aspects of the Research Network, enjoyment/alleviation of boredom, potential advantages of Research Networks to patients, and the feeling that the Network was, even if only locally, contributing to improvements in oral care.

\section{Belonging to a group}

The sense of isolation among GDPs was very apparent in the practitioners' comments, with almost all mentioning how important the Network had been in enhancing their sense of professional 'belonging,

'Involvement has been enjoyable, educational and constructive. It has been something new in my professional life that has introduced more variety to the sometimes mundane life of a general practitioner'.

The fact that the Network practitioners had motivation in common and formed a coherent group was clearly important to the vast majority of those participating. The importance of this group identity and shared goal shone through in the dentists' comments.

'Nice to be part of a larger community of dentists motivated in a similar fashion to myself'.

\section{'... a feeling of belonging to a body'.}

\section{Educational Aspects}

All the dentists participating had something positive to say about the educational experiences. This was described in detail for each of the workshops. However, the practitioners clearly felt they were benefiting, even if the subject matter of the workshops was complex.

'... an interesting experience, baffling at times, but ultimately very rewarding'.

\section{Enjoyment}

The usefulness of the Network for alleviating boredom and providing an enjoyable (though educational) experience was obviously an important aspect of the Network.

'The group activity is a welcome anti- dote to the isolation of general practice'

'I have enjoyed being part of the Dental Practice Network'

'It has been a great experience and most enjoyable'.

...an incredibly valuable forum?

\section{Potential advantages to patients}

However, perhaps the most important aspect of the practitioners' thoughts about being in the Network was a somewhat unexpected finding but was a central theme in most of the practitioners' comments.

'Considering that GDPs carry out very large volumes of treatment and are responsible (as a group) for the dental care of millions of patients, it is wonderful that at long last they are being invited to get involved in research. There should be much more practice based research involving general dental practitioners'

'I hope that the Network will be able to move into further research projects, otherwise practice life appears to 'stand still' with no improvements taking place'.

Some of the respondents were more specific about the skills they had gained and what they had learnt which would advantage patients.

'It has made it possible for me ... to design questionnaires tailored to the needs of my patients and use the result to improve my approach to dentistry'.

'It encourages research in dental practice, the use of audit in practice and the results of the study influences treatments and appointment intervals'

\section{The future}

All of the involved practitioners wished the group to continue and wished to participate in further research. Two ideas particularly emanated from the feedback and associated discussions. Firstly, the practitioners were keen to compare the data which they had collected with equivalent data from other sites. Suggestions were that interesting comparison data could be collected from access centres and PDS capitation patients. For the future, the participants strongly hoped that they could conduct a longitudinal study of their own patients, using the patient questionnaire used in the Network Research. Doing so would fulfil the third aim of the study they originally planned, ie to measure the effect of dental care on their patients' self-perceived health and well-being

\section{DISCUSSION}

The practice of dentistry changes rapidly. What drives the changes is debatable but it is perhaps optimistic to assume that it is the research generated from the academic centres which changes what dentists do with their patients on a day-to-day basis. Since the practice of dentistry depends so heavily on the interaction between the practitioner's knowledge base and the patient's value system, ${ }^{8}$ it seems essential that research about dental practice is undertaken in dental practices by dental practitioners with dental practice patients. It is in fact difficult to imagine any other setting in which one could undertake research which would have direct relevance and salience to general dental practitioners and their patients. This argument assumes that research is of value because, in the long term, it will benefit patients.

However, for practice-based research to develop and provide evidence which would truly act as a guide to dentists, practitioners must develop research skills as well as being research aware. It will only be when all those dental practitioners who wish to do research are equipped with the appropriate skills and support, that the research agenda will be truly primary care led. Arguably, if that were the case, research would also be of more direct relevance to general practice patients. Using practice research networks as a means of increasing true patient involvement in research may be an important step forward.

It has been suggested in a number of fora that primary dental care should NOT form its own research networks but should be part of 'primary care' as a whole and that dentists should form networks with medics, nurses and other health professions. Since remarkably few dental networks have come into being and even fewer dentists are involved in other primary care networks, it is difficult to gauge which model is preferable. Perhaps the ideal would be for both systems to run in parallel.

An important role which research networks play was highlighted by the practitioners in this group. The participating dentists derived immense personal and professional fulfilment from being part of a group with a common aim. The "networking' aspect of the project was at least as important as the research although both aspects were uniquely interdependent. The dentists' sense of enjoyment and satisfaction derived from 'belonging to a body' is an important finding. Demotivation of practitioners and consequent 
retiral from the profession leaves younger practitioners without role models and older ones with an increasing sense of isolation. Whilst many bodies exist to bring dentists together, funding practitioners for daytime meetings clearly enhanced their ability to enjoy and benefit from the learning process. This model of establishing shared goals and allowing participants to shape, and have active involvement in directing, their own professional development, is perhaps one which may be useful for those responsible for CPD courses.

The practitioners involved in the network came to it with many research ideas. It was they who felt that in order to be able to develop ideas, they needed to develop their research capabilities. The workshops were therefore designed to be driven by the practitioners' interest in addressing their own research agendas. As one set of skills were gained, this led to a heightened awareness among the group that some other skill was also needed. For example, the group gained skills in, and a theoretical knowledge of, questionnaire development but were unsure about the applicability of the measurement instrument in their practices. Rather than explore this in a didactic way, the group solved their own dilemma by pilot testing the instrument and then designing research for which it was applicable. This 'learning by doing' seemed to be an extraordinarily successful tactic. It was particularly useful with regard to data manipulation and analysis (Workshop 5). This workshop was extremely popular and led to several of the group being keen to purchase statistical analysis software packages.

The research network is now sadly coming towards the end of its 2 years of funding. Publicly funded projects such as this must be accountable. Whether this Dental Practice Network was 'successful' is a value judgement. However, evaluation of other networks has included output measures such as numbers of papers (in this case two are to be published) and process measures, such as numbers of research meetings (in this case six). However, as networking also results in less tangible but nevertheless important benefits, such as the development of informal, trust-based relationships, the quality of the experience for those involved is of paramount importance. The qualitative evaluation described in this paper is hopefully testament to the fact that the Research Network is a very worthwhile experience for all involved.

1. Laurence C O, Beilby J J, Morley J F, Newbury J, Wilkinson D, Symon B. Establishing a practice based primary care research network. Aust Fam Physician 2001; 30: 508-512.

2. Wassermdan R C, Slora E J, Bocian A B, Pleny G V Baker A E, Redlow SE, Kessel W. A national practicebased research network to improve children's health care. Paediatrics 1998; 102: 1350-1357.

3. Kreher N F, Sesnay J W. Accuracy of on-site data entry in a rural primary care network. Fam Med 1995; 27: 457-459.

4. Lattimer $\mathrm{V}$, Smith $\mathrm{H}$, Hungin $\mathrm{P}$, Clasper A, George S. Future provision of out-of-hours, primary medical care: a survey with two general practitioner networks. BrMed J 1996; 312:352-356.

5. Whitcomb C, Enzmann G, Pershadsingh HA, Johnson $R$, Ciuryla V, Reisin E. A comparison of Nisoldipine and amlodipine for the treatment of mild to moderate hypertension. Int J Clin Prac 2000; 54: 509-513.

6. Swartz L M, Woloshin S, Wasson J H, Renfrew R A Welch $\mathrm{H}$ G. Setting the revisit interval in primary care. J Gen Intern Med 1999; 14: 230-235.

7. Mold JW, Berlon E D. OAFP starts practice-based resource/research network. J Okla State Med Assoc 1996; 89: 433-434

8. Steele R S, Sesney J W, Kreher N E. The prevalence of alcohol abuse and dependence in two geographically distinct regions in Michigan: an UPRNet study. Wincons Med J 1999; 98: 54-57. 